TITLE:

\title{
Visualization of trapped charges being ejected from organic thin- film transistor channels by Kelvin- probe force microscopy during gate voltage sweeps
}

\section{$\operatorname{AUTHOR}(S)$ :}

Yamagishi, Yuji; Kobayashi, Kei; Noda, Kei; Yamada, Hirofumi

\section{CITATION:}

Yamagishi, Yuji ... [et al]. Visualization of trapped charges being ejected from organic thinfilm transistor channels by Kelvin-probe force microscopy during gate voltage sweeps. Applied Physics Letters 2016, 108(9): 093302.

\section{ISSUE DATE:}

2016-03-02

\section{URL:}

http://hdl.handle.net/2433/216683

\section{RIGHT:}

Copyright (year) AlP Publishing. This article may be downloaded for personal use only. Any other use requires prior permission of the author and AlP Publishing. The following article appeared in (Appl. Phys. Lett. 108, 093302 (2016); http://dx.doi.org/10.1063/1.4943140) and may be found at

http://scitation.aip.org/content/aip/journal/apl/108/9/10.1063/1.4943140; The full-text file will be made open to the public on 2 March 2017 in accordance with publisher's 'Terms and Conditions for Self-Archiving'. 


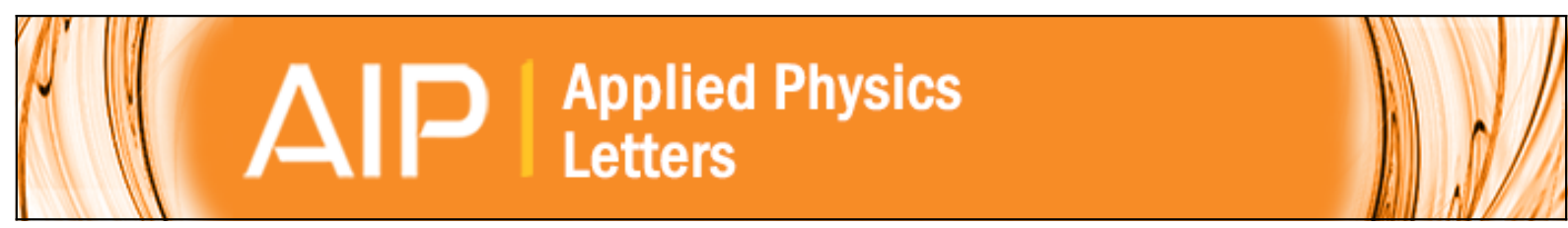

\section{Visualization of trapped charges being ejected from organic thin-film transistor channels by Kelvin-probe force microscopy during gate voltage sweeps}

Yuji Yamagishi, Kei Kobayashi, Kei Noda, and Hirofumi Yamada

Citation: Applied Physics Letters 108, 093302 (2016); doi: 10.1063/1.4943140

View online: http://dx.doi.org/10.1063/1.4943140

View Table of Contents: http://scitation.aip.org/content/aip/journal/apl/108/9?ver=pdfcov

Published by the AIP Publishing

\section{Articles you may be interested in}

Trapped charge mapping in crystalline organic transistors by using scanning Kelvin probe force microscopy Appl. Phys. Lett. 105, 193303 (2014); 10.1063/1.4901946

Analyzing the influence of negative gate bias stress on the transconductance of solution-processed, organic thinfilm transistors

J. Appl. Phys. 116, 074507 (2014); 10.1063/1.4893317

Influence of film structure and light on charge trapping and dissipation dynamics in spun-cast organic thin-film transistors measured by scanning Kelvin probe microscopy

Appl. Phys. Lett. 100, 263305 (2012); 10.1063/1.4720063

Independently driven four-probe method for local electrical characteristics in organic thin-film transistors under controlled channel potential

Rev. Sci. Instrum. 82, 093902 (2011); 10.1063/1.3637489

Increase in carrier mobility of organic ultrathin-film transistor with increasing molecular layers investigated by Kelvin probe force microscopy

J. Appl. Phys. 97, 124503 (2005); 10.1063/1.1937474

\section{Pure Metals • Ceramics Alloys • Polymers in dozens of forms}
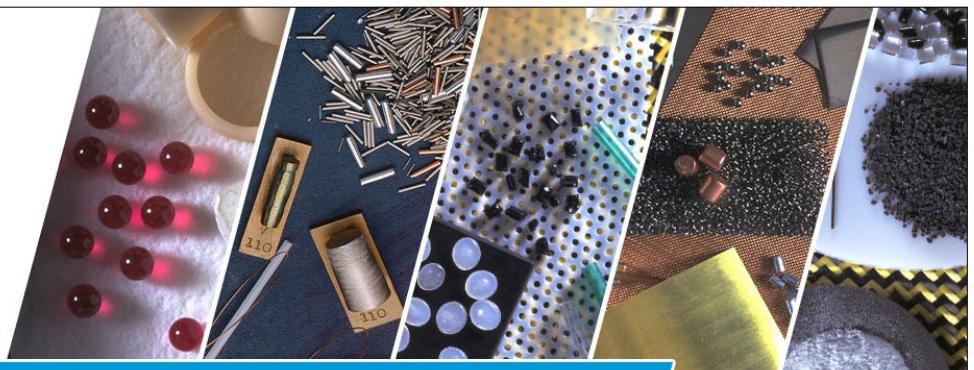

Small quantities fast Expert technical assistance $5 \%$ discount on online orders

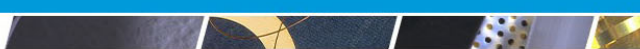




\title{
Visualization of trapped charges being ejected from organic thin-film transistor channels by Kelvin-probe force microscopy during gate voltage sweeps
}

\author{
Yuji Yamagishi, ${ }^{1}$ Kei Kobayashi, ${ }^{1,2, a)}$ Kei Noda, ${ }^{3}$ and Hirofumi Yamada ${ }^{1}$ \\ ${ }^{1}$ Department of Electronic Science and Engineering, Kyoto University, Kyoto 615-8510, Japan \\ ${ }^{2}$ The Hakubi Center for Advanced Research, Kyoto University, Kyoto 615-8520, Japan \\ ${ }^{3}$ Department of Electronics and Electrical Engineering, Keio University, Yokohama 223-8522, Japan
}

(Received 10 December 2015; accepted 20 February 2016; published online 2 March 2016)

\begin{abstract}
Kelvin-probe force microscopy (KFM) has been widely used to evaluate the localized charge trap states in the organic thin-film transistor (OTFT) channels. However, applicability of the KFM has been limited to the trapped charges whose lifetime is typically longer than several minutes because of the temporal resolution of the KFM. Therefore, it has not long been employed for studying the dynamics of the trapped charges in the OTFTs. Here, we demonstrate a method to visualize the transient distribution of the trapped charge carriers in operating OTFTs. The method allows visualizing the dynamics of the trapped charges during the gate voltage sweeps on a time scale of several hundreds of milliseconds. The experimental results performed on dinaphtho[2,3-b:2', $\left.3^{\prime}-\mathrm{f}\right]$ thieno[3,2-b]thiophene (DNTT) OTFTs indicate that, immediately after a bias voltage applied to a device was turned off, the primary discharging of the channel region around the electrode edges started and it limited the ejection process of the remaining accumulated charges to the electrodes, resulting in an increased density of long-lived trapped charges in a region distant from the electrodes. The presented results suggest that the method is useful to study the electrical connections at the interface between the DNTT grains and electrodes, or those between the grains. (C) 2016 AIP Publishing LLC. [http://dx.doi.org/10.1063/1.4943140]
\end{abstract}

Organic thin-film transistors (OTFTs) have been intensively studied for the development of printable electronics and flexible electronics. One of the critical issues for the practical applications of the OTFTs is the stability and reliability of the devices. Since the device parameters, such as the field-effect mobility and threshold voltage, are often affected by the trapped charges in the channel, ${ }^{1,2}$ characterization of the charge trap states is becoming very important. Among various experimental techniques that have been reported to be useful for the evaluation of the charge trap states of the OTFTs, ${ }^{3-5}$ the scanning probe techniques are very powerful in terms of spatial resolution. The electrostatic force microscopy (EFM) has been shown to be useful to probe microscopic nature of the trapped charges. ${ }^{6,7}$ Recently, the measurement of dynamic processes of charge carriers on sub-millisecond time scales in organic photovoltaic systems has been demonstrated by using the EFM. ${ }^{8}$ However, the derivation of the physical quantities such as the trapped charge density is not straightforward in the EFM. On the other hand, the Kelvin-probe force microscopy (KFM) allows us to quantitatively measure the surface potential in the OTFT channels, from which the trapped charge density can be easily estimated. ${ }^{9-11}$ In previous KFM experiments on the charge trapping in OTFTs, the distribution of the carriers trapped in deep states whose lifetime is comparable or longer than the measurement time was studied. ${ }^{12-16}$ This is because the surface potential mapping by the KFM typically takes more than several tens of seconds or several minutes, and thus, it is hard to evaluate transient

a)Electronic mail: keicoba@iic.kyoto-u.ac.jp phenomena that take place on a faster time scale. Recently, an alternative technique similar to KFM that can visualize the trapped charges through the measurement of the local threshold voltage by applying an ac modulation voltage to the gate electrode was reported by Ando et al. ${ }^{17}$ Since the OTFT device is in the accumulation regime during the gate voltage modulation during this method, the measured trapped charge distribution represents that during the device operation. Therefore, it has been difficult to visualize the dynamics of the trapped charges even with this method. We now propose a technique that allows us to visualize the dynamics of the trapped charges being ejected from the OTFT channels based on the KFM measurements during the gate voltage sweeps. We demonstrate the visualization of inhomogeneously distributed trapped charges being ejected from an OTFT channel of dinaphtho[2,3-b:2', $3^{\prime}$-f] thieno[3,2-b]thiophene (DNTT) and present the procedure to quantitatively estimate the charge densities ejected from the channel on two different time scales, namely, several hundred milliseconds and several seconds. We then discuss the origin of the inhomogeneous transient distribution of the trapped charge carriers.

Figure 1 shows a schematic of the experimental setup for the KFM measurements of a DNTT OTFT. We fabricated a DNTT OTFT with a bottom-contact-bottom-gate configuration as follows. We first patterned a pair of electrodes ( $20 \mathrm{~nm}$-thick $\mathrm{Au}$ on $2 \mathrm{~nm}$-thick $\mathrm{Cr}$ ) separated by a $2 \mu \mathrm{m}$ gap by photolithography on a heavily doped n-type $\mathrm{Si}$ wafer covered with a thermally grown oxide layer of $300 \mathrm{~nm}$ thickness. After treatment of the $\mathrm{SiO}_{2}$ surface with octadecyltriethoxysilane, a $50 \mathrm{~nm}$-thick DNTT layer was deposited in a vacuum 


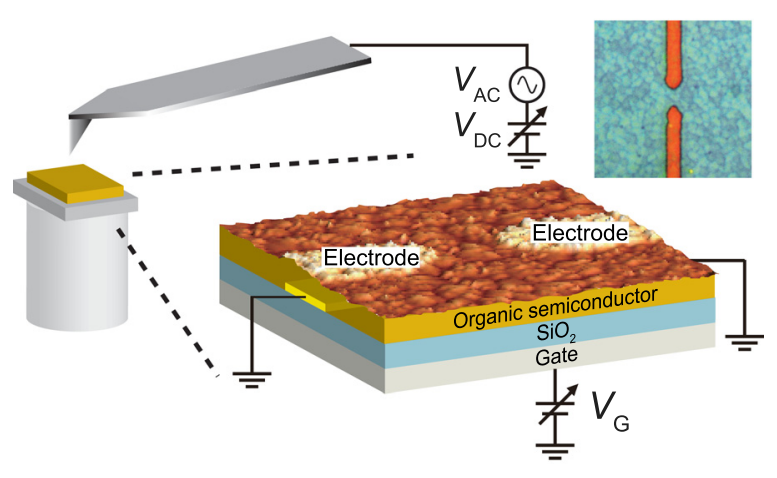

FIG. 1. Schematic of the experimental setup for KFM measurement of a DNTT OTFT. The inset shows an optical micrograph of the DNTT OTFT.

by thermal evaporation. The inset of Fig. 1 shows an optical micrograph of the DNTT OTFT. The width of the electrodes was about $2 \mu \mathrm{m}$. The KFM measurements were performed in a vacuum environment $\left(<8 \times 10^{-3} \mathrm{~Pa}\right)$ using a modified commercial AFM instrument (JEOL JSPM-5200) with a homebuilt scanning controller based on LabVIEW (National Instruments) and home-built electronics. The Pt-coated silicon cantilevers (Olympus OMCL-AC240TS) with a nominal spring constant of $2 \mathrm{~N} / \mathrm{m}$ and a resonance frequency of $70 \mathrm{kHz}$ were employed. The tip-sample distance was regulated in the constant frequency shift mode $(\Delta f=-20 \mathrm{~Hz})$ while the cantilever was self-oscillated with an amplitude of $20 \mathrm{~nm}$. A modulation voltage $(2 \mathrm{Vp}-\mathrm{p}, 1 \mathrm{kHz})$ was applied, and the frequency shift modulation was detected by a home-built phase-locked loop circuit and a lock-in amplifier (Zurich Instruments HF2LI). A bias voltage generated by the KFM feedback circuit that compensates for the contact potential difference between the tip and the sample, which was recorded as the surface potential $\left(V_{\mathrm{SP}}\right)$, was also applied to the tip.

Before we describe the details of the method based on the KFM measurements during the gate voltage sweeps, we measured the decay characteristics of the trapped charges in the OTFT channel. Figure 2(a) shows the time evolution of $V_{\mathrm{SP}}$ measured at a point on the DNTT layer approximately $8 \mu \mathrm{m}$ away from the electrode edge. For $t=0 \mathrm{~s}$ to $31 \mathrm{~s}$, all the electrodes were electrically grounded, and the $V_{\mathrm{SP}}$ was about $0.7 \mathrm{~V}$, which is defined as $V_{0}$. When a voltage pulse $\left(V_{\text {pulse }}\right.$ $=-10 \mathrm{~V}$ ) with a duration of $1 \mathrm{~s}$ was applied to the gate electrode at $t=31 \mathrm{~s}$, the $V_{\mathrm{SP}}$ immediately dropped to about $0 \mathrm{~V}$. This is because the positive charge carriers were injected and the $V_{\mathrm{SP}}$ on the DNTT layer became almost equal to that of the source and drain electrodes. When the pulse was turned off at $t=32 \mathrm{~s}, V_{\mathrm{SP}}$ abruptly jumped to about $2.8 \mathrm{~V}$, which is defined as $V_{\max (m e a s)}$, then it exponentially decreased to the initial value $\left(V_{0}\right)$ with a decay time constant of about $15 \mathrm{~s}$. The difference between $V_{\text {max (meas) }}$ and $V_{0}$ is defined as $\Delta V$ $=V_{\max (\text { meas })}-V_{0}$. It was expected that the maximum value of $V_{\mathrm{SP}}\left(V_{\mathrm{max}}\right)$ immediately after turning off the pulse is $-V_{\text {pulse }}=10 \mathrm{~V}$, and the number density of the injected carriers during the voltage pulse can be estimated as $C_{\mathrm{ox}}\left(V_{\max }-V_{0}\right) / e$ $=6.6 \times 10^{11} \mathrm{~cm}^{-2}$, where $e$ is the elementary charge and $C_{\mathrm{ox}}$ is the capacitance per unit area of the gate dielectric. However, the maximum number density of the injected charges from the measured potential was estimated as $C_{\mathrm{ox}}\left(V_{\text {max (meas) }}-V_{0}\right) / e$ $=C_{\mathrm{ox}} \Delta V / e=1.6 \times 10^{11} \mathrm{~cm}^{-2}$. We performed the same
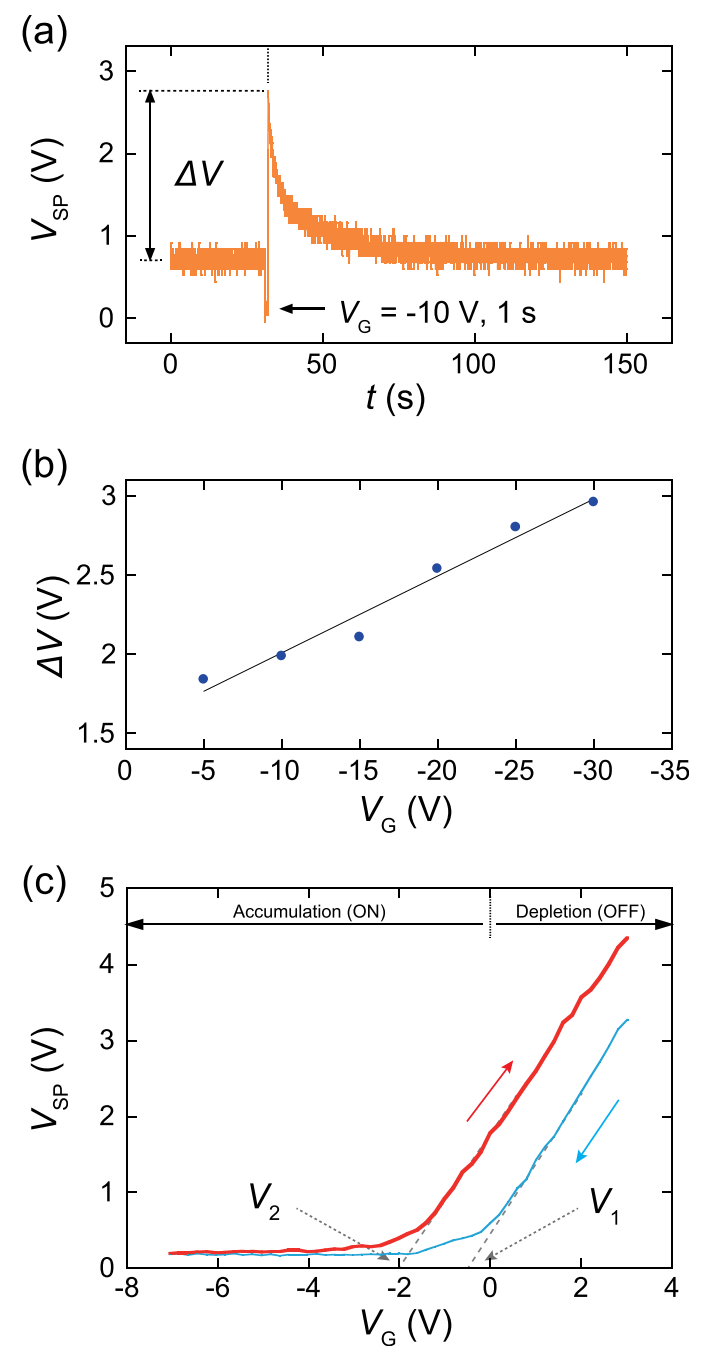

FIG. 2. (a) Temporal evolution of the $V_{\mathrm{SP}}$ measured in the channel region when a gate voltage pulse of $-10 \mathrm{~V}$ with the width of $1 \mathrm{~s}$ was applied at $t=31 \mathrm{~s} . \Delta V$ represents the difference between the initial $V_{\mathrm{SP}}$ and the potential measured just after the gate voltage was turned off. (b) $\Delta V$ measured versus gate voltage. (c) $V_{\mathrm{SP}}-V_{\mathrm{G}}$ characteristics measured in the channel region in different sweep directions.

measurements with different $V_{\text {pulse }}$ values ranging from $-5 \mathrm{~V}$ to $-30 \mathrm{~V}$. Figure 2(b) shows a plot of $\Delta V$ as a function of $V_{\text {pulse. }} \Delta V$ linearly correlates with $V_{\text {pulse, }}$ indicating that the density of the trapped charges is proportional to that of the injected carriers. As the absolute value of the slope of the fitted line $\left(4.8 \times 10^{-2}\right)$ is far lower than one, $\Delta V$ is considered to be associated with the number of charges in the relatively deep trap states whose lifetime is longer than the temporal resolution of the KFM measurement $(\sim 3 \mathrm{~ms})$. The result suggests that roughly $80 \%$ of the accumulated carriers diffused into the electrodes on a time scale shorter than a few milliseconds and the rest of the carriers could not smoothly diffuse to the electrodes because they were trapped in the trap sites in the channel.

We next measured the variation in $V_{\mathrm{SP}}$ during gate voltage $\left(V_{\mathrm{G}}\right)$ sweeps. Figure 2(c) shows a plot of $V_{\mathrm{SP}}$ for a $V_{\mathrm{G}}$ range between $-7 \mathrm{~V}$ and $3 \mathrm{~V}$. First, $V_{\mathrm{SP}}$ was recorded when $V_{\mathrm{G}}$ was swept from $3 \mathrm{~V}$ to $-7 \mathrm{~V}$ for $370 \mathrm{~ms}$ as shown as the thin (blue) curve in the figure. We define this direction of the $V_{\mathrm{G}}$ sweep as the forward sweep. $V_{\mathrm{SP}}$ was almost equal to $V_{\mathrm{G}}$ for $V_{\mathrm{G}}>0$, namely, the slope was almost equal to 1 , because the carriers in the channel were depleted. On the other hand, 
$V_{\mathrm{SP}}$ became almost equal to $0 \mathrm{~V}$ for $V_{\mathrm{G}}<0$, namely, the slope was almost 0 , because the carriers were injected and the variation in $V_{\mathrm{G}}$ was screened by the accumulated carriers. Second, $V_{\mathrm{SP}}$ was recorded when $V_{\mathrm{G}}$ was inversely swept from $-7 \mathrm{~V}$ to $3 \mathrm{~V}$ for $370 \mathrm{~ms}$ (backward sweep) as shown as the thick (red) curve. The deviation in the thick $V_{\mathrm{SP}}$ curve from the thin curve was caused by the positive charges that were not ejected to the source or drain electrode during the sweep, namely, the trapped charge whose lifetime is longer than the sweep time of $V_{\mathrm{G}}$. Thus, we can estimate the density of the positive trapped charges that remained after performing the $V_{\mathrm{G}}$ sweep from the lateral shift in the $V_{\mathrm{SP}}-V_{\mathrm{G}}$ curve. The shift can be calculated from the $V_{\mathrm{G}}$ values at which the two linear parts in the $V_{\mathrm{SP}}-V_{\mathrm{G}}$ curve intersect (local threshold voltage). The $V_{\mathrm{G}}$ values are defined as $V_{1}$ for the forward sweep and $V_{2}$ for the backward sweep. It is expected that we can visualize the dynamics of the trapped charges whose lifetime is longer than the $V_{\mathrm{G}}$ sweep time by measuring the local threshold voltage shift, $\Delta V_{\mathrm{th}}=V_{2}-V_{1}$, at each pixel in the channel region. Note that the $V_{\mathrm{SP}}-V_{\mathrm{G}}$ characteristics during the forward sweep should be started after the positive carriers in the channel are fully depleted, meaning that one has to wait for a decent time at each point, otherwise, the $V_{\mathrm{SP}}-V_{\mathrm{G}}$ curve would be shifted in the negative $V_{\mathrm{G}}$ direction and thus $\Delta V_{\text {th }}$ is misestimated. This means that in some cases, including the DNTT OTFT reported here, the total measurement time can be impractically long. However, it can be shortened if the variation of $V_{1}$ in the channel area is negligibly small and can be regarded as a constant. As $V_{1}$ represents the local threshold value in the depletion regime, we can estimate the variation of $V_{1}$ in the channel area by mapping $V_{\mathrm{SP}}$ in the channel while the device is in the depletion regime. In order to confirm this, we obtained a KFM image in the depletion regime and performed a histogram analysis for the $V_{\mathrm{SP}}$ in the channel region (see Fig. S1 in the supplementary material ${ }^{18}$ ). The $V_{\mathrm{SP}}$ in the channel region showed a Gaussian distribution with a mean value of $0.322 \mathrm{~V}$ and a standard deviation of 0.06 , which was very small. Thus, we assumed that $V_{1}$ in the channel region could be regarded as a constant and used $-0.322 \mathrm{~V}$ as the representative value.

The $V_{\mathrm{SP}}-V_{\mathrm{G}}$ characteristic measurements were performed on each pixel $(64 \times 64$ matrix $)$ of the OTFT channel while the topographic data were obtained on a $256 \times 256$ matrix. $V_{\mathrm{G}}$ was swept in a cyclic voltage sweep manner as shown as an inset in Fig. 3(a); starting from $0 \mathrm{~V}$ to $-10 \mathrm{~V}$ and then swept from $-10 \mathrm{~V}$ to $+10 \mathrm{~V}$, and returned to $0 \mathrm{~V}$ with the total sweep time of $1.47 \mathrm{~s}$. The $V_{\mathrm{SP}}-V_{\mathrm{G}}$ characteristics measured in a channel region and on a top electrode are presented in Fig. 3(a). The $V_{\mathrm{SP}}$ curve obtained on the electrode showed a gradual increase with an increase in $V_{\mathrm{G}}$, because $V_{\mathrm{SP}}$ is not only determined by the electrostatic force between a tip and the electrode, but also affected by the interaction between the cantilever and the channel area surrounding the electrode. On the other hand, the $V_{\mathrm{SP}}-V_{\mathrm{G}}$ characteristics measured in the channel showed a linear increase with a slope of 0.95 for the $V_{\mathrm{G}}$ greater than $V_{2}(\sim-2 \mathrm{~V})$.

Figure $3(\mathrm{~b})$ shows $V_{\mathrm{SP}}$ images corresponding to $V_{\mathrm{G}}$ $=-10 \mathrm{~V},-5 \mathrm{~V}, 0 \mathrm{~V}$, and $5 \mathrm{~V}$. The images were reconstructed from the data sets obtained from a series of $V_{\mathrm{SP}}-V_{\mathrm{G}}$
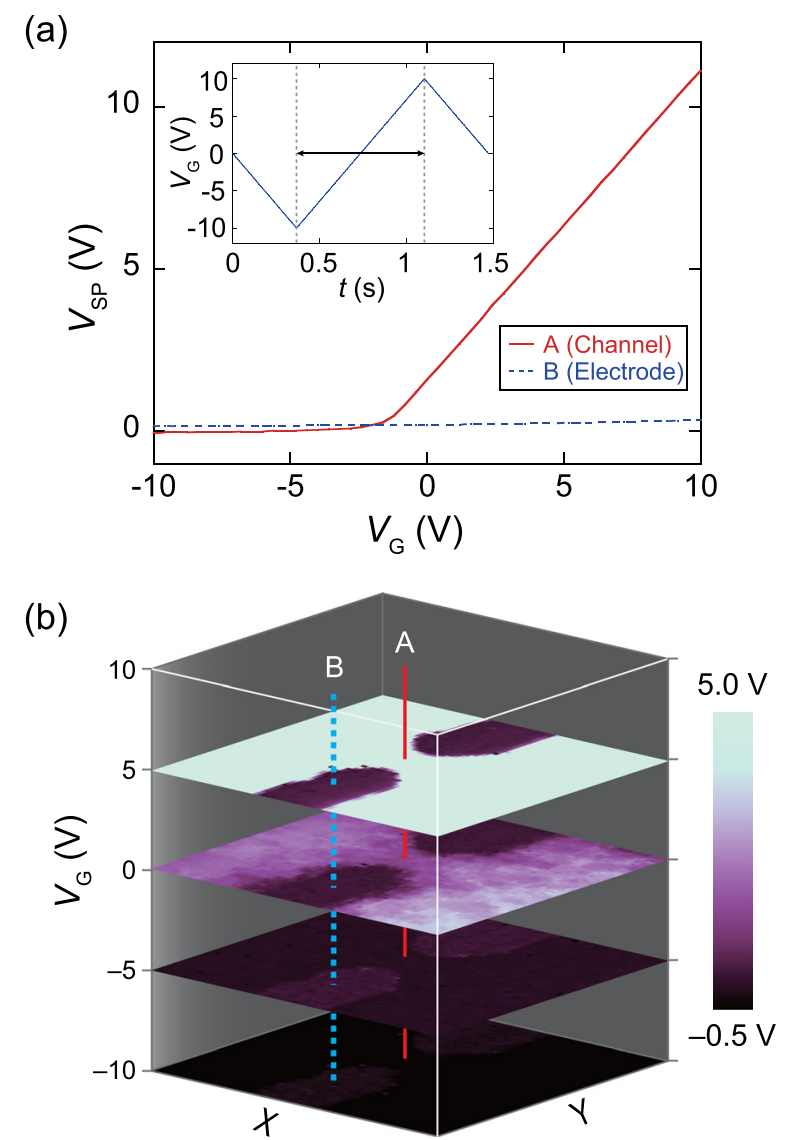

FIG. 3. (a) $V_{\mathrm{SP}}-V_{\mathrm{G}}$ characteristics measured in a channel region and on a top electrode. The inset shows the waveform of $V_{\mathrm{G}}$. The time span corresponding to the $V_{\mathrm{SP}}-V_{\mathrm{G}}$ measurement for calculating $V_{2}$ is indicated by the arrow. (b) Reconstructed surface potential images shown as a function of $V_{\mathrm{G}}$. Two vertical lines indicate the corresponding locations of the data sets shown in (a).

characteristic measurements performed on the DNTT OTFT. The locations at which curves $\mathrm{A}$ and $\mathrm{B}$ were recorded are indicated by the two vertical lines. As represented by the $V_{\mathrm{SP}}-V_{\mathrm{G}}$ characteristics shown in Fig. 3(a), $V_{\mathrm{SP}}$ in the channel was almost zero as well as that on the electrodes when $V_{\mathrm{G}}<0$. However, it started to linearly increase when $V_{\mathrm{G}}$ was greater than $V_{2}$, which was about $-2 \mathrm{~V}$ at location $\mathrm{A}$.

Figures 4(a) and 4(b) are a topographic image and a $\Delta V_{\text {th }}$ map, which were obtained by calculating the $V_{2}$ value of each $V_{\mathrm{SP}}-V_{\mathrm{G}}$ curve in the $V_{\mathrm{G}}$ range from $-10 \mathrm{~V}$ to $+10 \mathrm{~V}$, respectively. The topographic image exhibits many polycrystalline grains with the size of several hundred nanometers in the channel area. The remaining charge density was estimated at each pixel by calculating $C_{\mathrm{ox}} \Delta V_{\mathrm{th}} / e$, which is presented as the second scale bar in Fig. 4(b). In the $\Delta V_{\text {th }}$ map shown in Fig. 4(b), we observe a complicated contrast reflecting the features of the grain boundaries, which suggests that the grain boundaries largely disturb the carrier dissipation from the channel to the top electrodes. It is also noticeable that while $\Delta V_{\text {th }}$ is very low in the grains adjacent to the electrodes, it gradually increases with the increasing distance from the electrodes. This means that when the device was turned off, the charges accumulated in the grains near the top electrodes were quickly ejected, increasing the resistivity of the grains. This primary carrier ejection process 
(a)

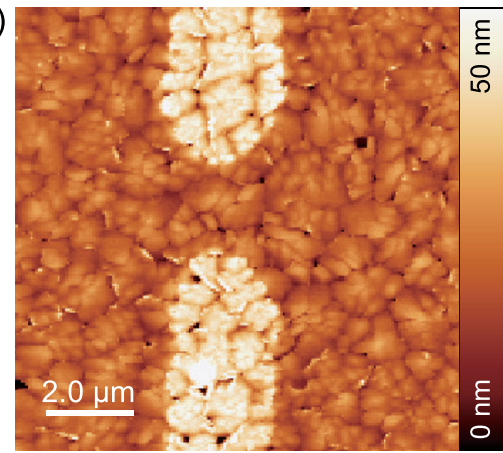

(b)

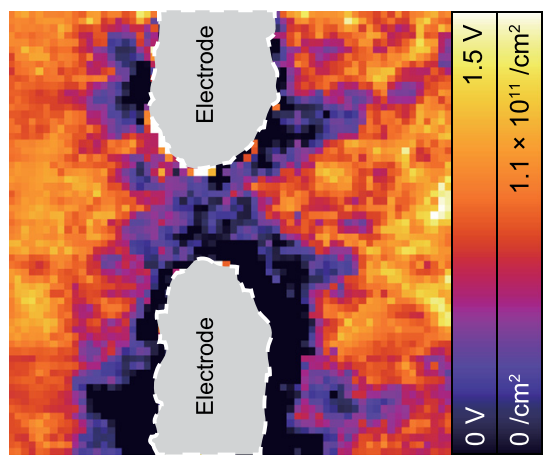

(c)

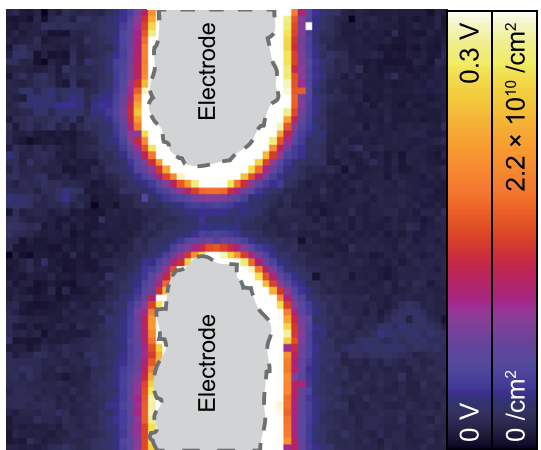

FIG. 4. (a) Topographic image, (b) $\Delta V_{\text {th }}$ map, and (c) slope $(S)$ map of the DNTT-OTFT. Charge density remaining after the voltage sweep was calculated as $C_{\mathrm{ox}} \Delta V_{\mathrm{th}} / e$, which is presented as the second scale bar in (b). Charge density being ejected during the sweep was calculated as $(1-S) C_{\mathrm{ox}}\left(V_{\mathrm{G}(\mathrm{max})}-V_{1}\right) / e$, which is presented as the second scale bar in (c).

increased the time needed for discharging the carriers in the grains far from the electrode and thus led to the gradual distribution of the trapped carriers. In the brightest area of the channel region in Fig. 4(b), roughly as many as $8 \%$ of the accumulated charges were trapped. These trapped carriers potentially give rise to an increase in the off-current and deterioration of the device stability. ${ }^{14}$ In order to decrease the density of the observed trapped charges, reducing the contact resistance between the electrodes and channel layer is effective because it shortens the time constant of the carrier ejection and thereby enables faster switching of the device.

We also analyzed the slope of the linear part in the reverse bias region of each $V_{\mathrm{SP}}-V_{\mathrm{G}}$ characteristic and obtained a slope map, as shown in Fig. 4(c). Contrary to the expectation that the slope should be one, the obtained values were slightly lower than one over the entire channel region. In principle, the slope $(S)$ deviates from 1 if the trapped charges were being ejected during the sweep. Therefore, we can roughly estimate the number density of the trapped charge being ejected during the sweep by calculating $(1-S) C_{\mathrm{ox}}\left(V_{\mathrm{G}(\max )}-V_{1}\right) / e$, where $V_{\mathrm{G}(\max )}$ is the maximum $V_{\mathrm{G}}$ for the backward sweep. This is also presented as the second scale bar in Fig. 4(c). In the channel region in proximity to the top electrodes, the slope gradually changes from zero to one as the distance from the electrodes increases. It is also noticeable that some contrasts observed in the slope map, or the map of the trapped charges during the sweep, seem to correspond to the shapes of the grains. The contrast observed in the slope map reflects the resistance between the corresponding channel region and the top electrodes, this is, to say, how the channel region is electrically connected to the top electrodes. Since the time constant of the charge dissipation is simply described by the product of the channel resistance and the gate capacitance based on a distributed constant circuit model when the contact resistance at the electrode/organic semiconductor is negligible, ${ }^{19,20}$ a lower channel resistance should result in a lower time constant and thus faster charge dissipation. Consequently, the values of the slopes in the grains, which are electrically well-connected to the electrodes, should be lower than those that are not connected. In other words, the number of trapped charges dissipated during the sweep is greater in the grains that are close to the electrodes.

As we have described, the technique presented here allows us to investigate the dynamics of the charges in the
OTFT channels on two different time scales. Although limited by the temporal resolution of the KFM measurement, the technique can be useful to study the charge dynamics during the ejection process and the injection process on time scales ranging from tens of milliseconds to a few minutes by changing the $V_{\mathrm{G}}$ sweep range and rate. We confirmed that the charge dynamics with different time scales can be measured by varying the bias sweep time (see Fig. S3 in the supplemental material ${ }^{18}$ ). As for spatial resolution, it is in principle the same as that of the conventional KFM, which is typically on the order of $10 \mathrm{~nm} \cdot{ }^{21-23}$ Therefore, by increasing the number of measurement points and limiting the scan area to the area of concern, we anticipate that we can visualize the dynamic processes in the organic thin films at a sub-grain scale resolution with our technique.

In summary, we demonstrated a method to visualize the temporal distribution of charge carriers trapped in the channel region during the carrier ejection process in the OTFTs. The obtained charge distribution map suggests that the carrier ejection process in the DNTT OTFT was limited by primary discharging of the channel around the electrode edges. The resulting highly resistive channel region increased the time constant of discharging of the channel and, consequently, led to an increased trapped charge density in the region far from the electrodes. We also showed that the method is also useful to characterize the electrical connections at the interface between the DNTT grains and electrodes, or those between the grains by analysis of a slope map. The method presented here is not only useful to study the carrier dynamics in the OTFTs but can be applied to visualize the dynamics of the surface potential on a time scale of several hundreds of milliseconds or longer.

This work was supported by Grant-in-Aid for Challenging Exploratory Research (Grant No. 25600097) from Japan Society for the Promotion of Science.

\footnotetext{
${ }^{1}$ H. Sirringhaus, Adv. Mater. 17, 2411 (2005).

${ }^{2}$ T. Hallam, C. M. Duffy, T. Minakata, M. Ando, and H. Sirringhaus, Nanotechnology 20, 025203 (2009).

${ }^{3}$ L.-L. Chua, J. Zaumseil, J.-F. Chang, E. C.-W. Ou, P. K.-H. Ho, H. Sirringhaus, and R. H. Friend, Nature 434, 194 (2005).

${ }^{4}$ C. Westermeier, M. Fiebig, and B. Nickel, Adv. Mater. 25, 5719 (2013).

${ }^{5}$ B. Rösner, N. Zeilmann, U. Schmidt, and R. H. Fink, Org. Electron. 15, 435 (2014).

${ }^{6}$ E. M. Muller and J. A. Marohn, Adv. Mater. 17, 1410 (2005).
} 
${ }^{7}$ M. Jaquith, E. M. Muller, and J. A. Marohn, J. Phys. Chem. B 111, 7711 (2007).

${ }^{8}$ R. Giridharagopal, G. E. Rayermann, G. Shao, D. T. Moore, O. G. Reid, A. F. Tillack, D. J. Masiello, and D. S. Ginger, Nano Lett. 12, 893 (2012). ${ }^{9}$ L. Bürgi, H. Sirringhaus, and R. H. Friend, Appl. Phys. Lett. 80, 2913 (2002).

${ }^{10}$ J. A. Nichols, D. J. Gundlach, and T. N. Jackson, Appl. Phys. Lett. 83, 2366 (2003).

${ }^{11}$ K. P. Puntambekar, P. V. Pesavento, and C. D. Frisbie, Appl. Phys. Lett. 83, 5539 (2003).

${ }^{12}$ M. Tello, M. Chiesa, C. M. Duffy, and H. Sirringhaus, Adv. Funct. Mater. 18, 3907 (2008).

${ }^{13}$ S. G. J. Mathijssen, M. Cölle, A. J. G. Mank, M. Kemerink, P. A. Bobbert, and D. M. de Leeuw, Appl. Phys. Lett. 90, 192104 (2007).

${ }^{14}$ S. G. J. Mathijssen, M. Kemerink, A. Sharma, M. Cölle, P. A. Bobbert, R. A. J. Janssen, and D. M. De Leeuw, Adv. Mater. 20, 975 (2008).

${ }^{15}$ L. C. Teague, O. D. Jurchescu, C. A. Richter, S. Subramanian, J. E. Anthony, T. N. Jackson, D. J. Gundlach, and J. G. Kushmerick, Appl. Phys. Lett. 96, 203305 (2010).
${ }^{16}$ L. C. Teague, M. A. Loth, and J. E. Anthony, Appl. Phys. Lett. 100, 263305 (2012).

${ }^{17}$ M. Ando, S. Heike, M. Kawasaki, and T. Hashizume, Appl. Phys. Lett. 105, 193303 (2014).

${ }^{18}$ See supplementary material at http://dx.doi.org/10.1063/1.4943140 for additional experimental results. In Fig. S1, we show the histogram analysis. In Fig. S2, we show how the bias stress effect observed in the $\Delta V_{\text {th }}$ image was calibrated. In Fig. S3, the $V_{\mathrm{SP}}-V_{\mathrm{G}}$ characteristics measured with different bias sweep times is presented.

${ }^{19}$ T. Miyadera, T. Minari, K. Tsukagoshi, H. Ito, and Y. Aoyagi, Appl. Phys. Lett. 91, 13512 (2007).

${ }^{20}$ B. H. Hamadani, C. A. Richter, J. S. Suehle, and D. J. Gundlach, Appl. Phys. Lett. 92, 203303 (2008).

${ }^{21}$ U. Zerweck, C. Loppacher, T. Otto, S. Grafström, and L. M. Eng, Phys. Rev. B 71, 125424 (2005).

${ }^{22}$ F. Krok, K. Sajewicz, J. Konior, M. Goryl, P. Piatkowski, and M. Szymonski, Phys. Rev. B 77, 235427 (2008).

${ }^{23} \mathrm{C}$. Li, S. Minne, Y. Hu, J. Ma, J. He, H. Mittel, V. Kelly, N. Erina, S. Guo, and T. Mueller, Bruker Application Note \#140, 2013. 